\title{
Desenvolvimento Psicológico na Fase Escolar de Crianças Nascidas Pré-termo em Comparação com Crianças Nascidas a Termo
}

\author{
Maria Beatriz Martins Linhares ${ }^{12}$ \\ Juliana Thomazatti Chimello \\ Maria Beatriz, Machado Bordin \\ Ana Emilia Vita Carvalho \\ Francisco Eulógio Martiner \\ Universidade de São Paulo, Ribeirão Preto
}

\begin{abstract}
Resumo
O presente estudo objetivou avaliar indicadores do desenvolvimento psicológico na fase escolar de crianças nascidas pré-termo com muito baixo peso e compará-los aos de crianças nascidas a termo, quanto às áreas intelectual, emocional e comportamental. A amostra foi composta por 40 crianças de 8 a 10 anos, subdivididas em 2 grupos: Pré-termo (PT), 20 crianças nascidas <37 semanas de idade gestacional e peso $\leq 1.500$ g e A Termo (AT), 20 crianças nascidas a termo com peso > 2.500g. Foram utilizados o Raven, o Desenho da Figura Humana e a Escala Comportamental Infantil. As crianças PT apresentaram mais freqüentemente nível intelectual inferior à média e problemas comportamentais em comparação às AT. Não houve diferença entre os grupos quanto aos aspectos emocionais. Os problemas de enurese, medo, tiques, impaciência e dificuldade de permanência nas atividades foram significativamente mais freqüentes no PT do que no AT. Verificou-se que, no grupo PT, quanto menor o nível intelectual das crianças, mais problemas de comportamento elas apresentavam.

Palavras-chave: Pré-termo; muito baixo peso de nascimento; desenvolvimento; inteligência; comportamento.
\end{abstract}

Psychological Development of School-age Children Born Preterm in Comparison with Children Born Full-Term

\begin{abstract}
The present study aims to assess psychological development in terms of behavioral, intellectual and emotional indicators of preterm and very low birthweight children at school age in comparison with full-term children. The sample in this study consisted of forty children aged from 8 to 10 years. They were divided into 2 groups: Preterm (PT) consisting of 20 children born $<37$ gestational weeks with a $\leq 1.500 \mathrm{~g}$ birthweight and Full term (FT) also consisting of 20 subjects who were born with a $>2.500 \mathrm{~g}$ birthweight. Raven's Special Scale, Draw-a-Person and Child Behavior Scale were used in the psychological assessment. PT group presented more behavioral problems and lower intellectual level when compared to the FT group. Enuresis, fear, tics, impatience and lack of focus on activities were significantly more frequent in the PT group than in the FT group. In the PT group the lower the intellectual level of the children the more behavior problems they presented.

Key-words: Preterm; very low birth weight; development; intelligence; behavior.
\end{abstract}

Na trajetória do desenvolvimento de crianças, o nascimento prétermo associado à condição de muito baixo peso constitui-se em fator adverso de alto risco neonatal que pode comprometer o desenvolvimento psicológico futuro da criança (Lewis, Duglokinski, Caputo \& Griffin, 1988). Os bebês nascidos com idade gestacional abaixo de 37 semanas e com peso de nascimento igual ou abaixo de 1.500 gramas necessitam de internação em Unidade de Tratamento Intensivo Neonatal (UTIN) para assegurar a sua sobrevivência. O tratamento médico dispensado ao neonato pré-termo durante os cuidados especializados na UTIN produzem, por sua vez, fatores adversos que causam desconforto e dor ao bebê, os quais podem ter impacto negativo no seu desenvolvimento psicológico a curto e médio prazo (Grunau, 2000).

Avanços significativos têm sido realizados quanto aos índices de sobrevivência de neonatos pré-termo de alto risco em UTIN, atingindo nos EUA taxas bem-sucedidas em torno de 50 a $90 \%$ em neonatos de 24 a 28 semanas de idade gestacional (Lorenz, 2000). Enfrentando as adversidades, muitas vezes sobrevivem

\footnotetext{
${ }^{1}$ Endereço para correspondência: Prof ${ }^{a}$. Dr ${ }^{\mathrm{a}}$. Maria Beatriz Martins Linhares. Av João Fiúza, 1725, 93, 14024 250, Ribeirão Preto, SP. Fone: (16) 6250309, 6250490, 6372702, Fax: (16)6250309,6250490.E-mail:linhares@fmrp.usp.br

${ }^{2}$ Apoio Financeiro: FAPESP/ CNPq/ FAEPA-HCFMRP
}

recém-nascidos com peso abaixo de 1.000 gramas, graças à combinação do suporte de avanços tecnológicos e científicos da área da Medicina e o poder de recuperação ou processo de resiliência alcançado por esses bebês na luta pela sobrevivência no ambiente protegido da UTIN. Taxas de sobrevivência em torno de $85 \%$ foram encontradas em bebês nascidos com peso igual ou menor a $1.500 \mathrm{~g}$ e internados na UTIN do Hospital das Clínicas da Faculdade de Medicina de Ribeirão Preto - USP (HCFMRP) no período de 2000 a 2003.

No entanto, considerando-se os possíveis efeitos adversos advindos do alto risco neonatal, verifica-se a necessidade de se avaliar o curso do desenvolvimento psicológico desses bebês, após a alta hospitalar. Torna-se necessário, portanto, o acompanhamento de crianças nascidas pré-termo em condição de vulnerabilidade biológica, a fim de identificar a presença tanto de dificuldades, quanto de recursos das crianças. Deste modo, pode-se obter informações sobre sua qualidade de vida para que possam ser implementadas medidas preventivas ou, se necessário, terapêuticas orientadas para a promoção do desenvolvimento psicológico.

No campo interdisciplinar da Psicologia Pediátrica, na área vinculada especificamente à Neonatologia, verificam-se estudos de seguimento longitudinal do desenvolvimento de neonatos pré- 
termo egressos de UTIN. A avaliação do desenvolvimento de crianças nascidas pré-termo de muito baixo peso em comparação com pares nascidos a termo têm sido objeto de vários estudos, que focalizam diversos marcos desenvolvimentais relevantes, como por exemplo, os primeiros anos de vida (Langkamp, Kim \& Pascoe, 1998; Maas \& cols., 2000), a fase pré-escolar (Callanan \& cols., 2001; Girouard \& cols., 1998) e a fase escolar (Naudeau, Boivin, Tessier, Lefebvre \& Robaey, 2001; Taylor, Klein, Schatschneider \& Hack, 1998).

Crianças nascidas pré-termo e com peso abaixo de $1.500 \mathrm{~g}$ apresentam significativamente mais dificuldades, quando comparadas a crianças nascidas a termo com peso igual ou acima de $2.500 \mathrm{~g}$, em diversas áreas do desenvolvimento. As primeiras apresentam, quando comparadas às segundas, rebaixamento intelectual (Damann \& cols., 1996; Waber \& McCornick, 1995), problemas emocionais (LevyShiff \& cols., 1994) e comportamentais (Linhares \& cols., 2000; Nadeau \& cols., 2001; Stjernqvist, 1993).

As seqüelas neurológicas observadas no período neonatal ou em etapas posteriores do desenvolvimento (Antunha, 1994; Brazelton, 1994), assim como as condições ambientais familiares da criança (Bradley \& cols., 1993; Liaw \& Brooks-Gunn, 1993) atuam de modo significativo a atenuar ou agravar o impacto do risco da prematuridade e do muito baixo peso ao nascer no desenvolvimento da criança. Quanto mais baixo o peso ao nascer e quanto mais risco psicossocial presente no ambiente familiar, mais dificuldades podem ocorrer na trajetória de desenvolvimento da criança (Bordin, Linhares \& Jorge, 2001; Piecuch, Leonard, Cooper \& Sehring, 1997).

Esses achados revestem-se de grande preocupação na medida em que a presença de vários fatores de risco na vida de uma mesma criança tende a resultar em um efeito multiplicador da adversidade no desenvolvimento, constituindo-se, portanto em condição de "múltiplo risco". Segundo Rutter (1987), mecanismos de proteção ao desenvolvimento necessitam ser ativados sempre que se detectar uma cadeia de eventos negativos em trajetórias de desenvolvimento, a qual torna o indivíduo vulnerável.

$\mathrm{Na}$ fase escolar, as tarefas desenvolvimentais normativas definem a expectativa de competência cognitiva para o adequado aprendizado formal escolar e de competência comportamental de auto-regulação e adaptação às demandas do meio social ampliado. Nesse sentido, os estudos de seguimento de crianças nascidas pré-termo na fase escolar têm por objetivo avaliar áreas relevantes do desenvolvimento psicológico e vinculadas a esse marco desenvolvimental, tais como a área intelectual, emocional ou comportamental.

Taylor e colaboradores (1998) examinaram preditores do desempenho de crianças nascidas com muito baixo peso $(<1.500 \mathrm{~g})$ na fase escolar, quanto à função cognitiva, habilidades neuropsicológicas, desempenho acadêmico e comportamento. Os achados mostram que, mesmo controlando-se os fatores de risco sócio-econômico, o risco neonatal influenciou o desempenho cognitivo e as habilidades neuropsicológicas das crianças avaliadas. Quanto maior o risco neonatal (hemorragia intracraniana, septicemia, apnéia, enterocolite necrosante, doença pulmonar crônica) maior foi o nível de deficiência do desenvolvimento avaliado na fase escolar.

O estudo de Naudeau e colaboradores (2001) acerca do desenvolvimento de crianças aos 5 e 7 anos nascidas com menos de
29 semanas de idade gestacional e $1.500 \mathrm{~g}$, comparadas a crianças nascidas a termo, verificou a correlação entre variáveis neonatais e desempenho intelectual e neuromotor. Além disso, os autores encontraram que a extrema prematuridade tinha um efeito indireto no comportamento da criança através do atraso intelectual e neuromotor. As crianças nascidas com risco neonatal devido à prematuridade apresentaram nível cognitivo mais rebaixado e mais problemas de isolamento, desatenção e hiperatividade do que as crianças nascidas a termo.

Se de um lado, a literatura internacional é bastante produtiva em relação a essa temática, por outro lado, no cenário brasileiro poucos estudos dedicam-se a investigar a trajetória de desenvolvimento das crianças nascidas com muito baixo peso, especialmente a médio ou longo prazo. No âmbito da pesquisa nacional, alguns pontos merecem destaque para serem investigados. Primeiramente, são necessários estudos sobre a avaliação do desenvolvimento das crianças nascidas pré-termo na fase escolar, após a passagem do enfrentamento de tarefas evolutivas ou marcos significativos do desenvolvimento. Em segundo lugar, necessita-se de estudos que focalizem na avaliação psicológica no conjunto das dimensões intelectual, emocional e comportamental, a fim de avaliar correlatos do desenvolvimento relevantes para o enfrentamento adaptativo às demandas de aprendizagem e de relacionamento interpessoal presentes na vida da criança na fase escolar. Em terceiro lugar, são necessários estudos do tipo caso-controle, que inclui a análise de comparação entre grupos de crianças com risco neonatal da prematuridade emparelhadas a crianças sem risco.

Os comprovados efeitos adversos dos fatores da prematuridade e do muito baixo peso ao nascer sobre o desenvolvimento, assim como a escassez de pesquisas nacionais de seguimento, justificam a realização do presente estudo. Inserido em um projeto mais amplo este estudo teve por objetivo: a) avaliar o nível intelectual e indicadores do funcionamento adaptativo emocional e comportamental de crianças na fase escolar nascidas pré-termo com muito baixo peso $(\leq 1.500 \mathrm{~g})$, comparadas a crianças nascidas a termo e com peso de nascimento acima de $2.500 \mathrm{~g}$; b) verificar a relação entre nível intelectual e problemas emocionais e de comportamento, em nível clínico, no grupo de crianças nascidas pré-termo com muito baixo peso.

Destaca-se que a presente investigação teve a intenção de ser conduzida com amostra de crianças que se encontravam em condição de "múltiplo risco", ou seja, que apresentassem na sua história de vida a presença de risco biológico da prematuridade, assim como de risco psicossocial, devido a pertencerem a famílias com poucos recursos educacionais e econômicos, atendidas em serviços públicos das áreas de Saúde e Educação.

\section{Método}

\section{Participantes}

A amostra foi composta por 40 crianças de 8 a 10 anos de idade (62\% de meninas) distribuídas em dois grupos, emparelhados quanto a gênero e faixa etária das crianças, e balanceados quanto a escolaridade e qualificação ocupacional 
das mães. O grupo Pré-termo (PT) era composto por 20 crianças nascidas pré-termo (idade gestacional média de 29 semanas $[d p= \pm 3])$ e com peso de nascimento igual ou abaixo de 1.500 gramas (peso médio de $1.150 \mathrm{~g}[d p= \pm 270]$ ). O grupo PT incluía $65 \%$ de crianças em risco neonatal moderado ( $<5$ pontos) e $35 \%$ em risco grave ( $\geq 5$ pontos) segundo a Clinical Risk Index for Babies (CRIB) (Cockburn \& cols., 1993). As crianças deste grupo nasceram no Hospital das Clínicas da Faculdade de Medicina de Ribeirão Preto (HCFMRP), o qual caracteriza-se por ser um hospital geral universitário, de nível terciário, vinculado ao Sistema Único de Saúde (SUS). O grupo A Termo (AT), por sua vez, era composto por 20 crianças, alunos regulares de uma escola pública estadual de Ribeirão Preto, nascidas a termo e com peso de nascimento superior a 2.500 gramas.

Para a composição da amostra do grupo P'T foram utilizados os livros anuais de nascimentos do Setor de Neonatologia da Pediatria do HCFMRP. Primeiramente, foram identificadas 252 crianças nascidas com peso igual ou inferior a $1.500 \mathrm{~g}$ e obtidos os endereços residenciais das mesmas. Foram selecionados os casos que residiam em Ribeirão Preto e região administrativa e excluídos os casos de óbito. Obteve-se um total de 127 crianças elegíveis para o estudo, das quais 37 compareceram para o estabelecimento do contrato de participação. A aceitação em participar do estudo não garantiu, no entanto, a participação de todas as crianças, visto que oito crianças não compareceram na primeira sessão agendada para o início da avaliação e nove crianças abandonaram a mesma. Portanto, foram avaliadas 20 crianças do grupo Pré-Termo, sendo 65\% de meninas.

Para a composição da amostra do grupo AT foram distribuídas uma ficha de caracterização de variáveis da criança e de seus pais e a Escala Comportamental Infantil A2 de Rutter (ECI) a todos os 500 alunos de $2^{a}$ a $4^{a}$ séries de uma escola estadual de Ribeirão Preto. Das 354 crianças que devolveram as escalas e as fichas preenchidas, foram selecionadas todas aquelas de 8 a 10 anos de idade, nascidas com peso superior a 2.500 gramas, cujas fichas e escalas estivessem preenchidas pelas mães e de forma completa. A partir desta pré-seleção, foram obtidas 180 crianças elegíveis para constituir o grupo AT. Foram então aplicados os critérios de emparelhamento dos grupos por gênero e faixa etária e balanceamento do nível de escolaridade e qualificação ocupacional das mães, obtendo-se a amostra final de 20 crianças ( $60 \%$ de meninas) com as características sócioeducacionais familiares mais próximas possíveis às das crianças do grupo PT.

\section{Aspectos Éticos}

O presente estudo contou com a aprovação do Comitê de Ética em Pesquisa do HCFMRP. As familias receberam informações sobre o estudo e tinham liberdade de participar ou não do mesmo. Caso as famílias quisessem desistir de participar do estudo poderiam fazê-lo a qualquer momento. Foram assegurados o sigilo da identificação dos participantes e o ressarcimento dos gastos com transporte para as familias do grupo PT compareceram ao hospital para a coleta dos dados. As familias que concordaram em participar assinaram um termo de consentimento livre e esclarecido. Da mesma forma procedeu-se com o grupo AT, após a exposição dos objetivos do presente estudo aos coordenadores da escola estadual e obtenção de sua aprovação para a realização do estudo na escola. Os responsáveis pelas crianças assinaram o termo de consentimento livre e esclarecido. Todas as famílias receberam devolutivas das avaliações realizadas.

\section{Instrumentos e Medidas}

Foram utilizados os seguintes instrumentos: a) Matrizes Progressivas Coloridas de Raven - Escala Especial com padronização brasileira de Angelini, Alves, Custódio e Duarte (1987); b) Escala Comportamental Infantil A2 de Rutter (ECI), traduzida, adaptada e padronizada por Graminha (1998); c) Desenho da Figura Humana de Goodenough (DFH), com padronização brasileira de Hutz e Antoniazzi (1995); d) Ficha de caracterização de variáveis das crianças e de seus pais; e) Prontuários médicos do HCFMRP.

\section{Procedimento}

A coleta de dados do grupo PT ocorreu no HCFMRP, enquanto a do grupo AT ocorreu na escola. A avaliação das crianças do grupo PT foi realizada em duas sessões, previamente agendadas. A $1^{\text {a }}$ sessão foi destinada ao preenchimento da ficha de caracterização dos participantes e à aplicação dos testes psicométricos Raven e DFH. A $2^{a}$ sessão foi destinada à avaliação do comportamento da criança, segundo a opinião das mães, através da ECI. Além disso, foi realizada consulta e análise documental dos prontuários médicos das crianças, a fim de se obter informações sobre as condições neonatais registradas pelo pediatra por ocasião do nascimento no HCFMRP.

A coleta de dados do grupo AT, por sua vez, incluiu primeiramente a distribuição da ECI na classe escolar, de modo que cada criança levava o termo de consentimento, uma ficha de caracterização e a escala ECI para casa, a fim de que suas mães respondessem conforme as instruções impressas nos mesmos. As mães deveriam devolver o material preenchido à professora, por meio do (a) filho (a). Em seguida, os testes de Raven e DFH foram aplicados individualmente em cada uma das 20 crianças selecionadas de acordo com os critérios de composição do grupo AT.

\section{Análise dos Dados}

O estudo teve um delineamento transversal e de comparação entre dois grupos independentes. Em uma primeira etapa, os dados foram submetidos à preparação para análise. Os testes psicométricos de Raven e DFH foram avaliados de acordo com as normas dos respectivos manuais de aplicação. O desempenho intelectual das crianças foi analisado quanto ao percentil no teste de Raven. No DFH, a partir da pontuação de itens específicos, foram obtidos os percentis referentes aos itens evolutivos e indicadores emocionais, respectivamente. No caso da avaliação 
dos itens evolutivos, quanto maior o número de itens evolutivos, mais alto o percentil, sugerindo melhor nível intelectual da criança. Quanto aos itens emocionais, quanto maior o número de itens, mais baixo o percentil, sugerindo a presença de mais indicadores de problema de ordem emocional. Os percentis nos testes relativizam o escore bruto em relação à amostra de padronização, respeitando a faixa de idade cronológica. No tratamento dos dados os percentis foram considerados como os escores obtidos pelas crianças no teste.

$\mathrm{Na}$ avaliação da ECI, em primeiro lugar, foi atribuída a cada item uma pontuação de zero a dois, considerando-se: valor zero (ausente), os itens que na avaliação da mãe nunca se aplicavam a seu filho; valor um (moderado), os itens que se aplicavam ocasionalmente; valor dois (grave), os itens que se aplicavam com certeza. Em segundo lugar, foi calculada a pontuação total na escala. Em terceiro lugar, a pontuação total de cada criança foi comparada com a nota de corte do nível clínico, segundo padronização de Graminha (1998). De acordo com esta, o escore maior do que 16 pontos indica a existência de problemas comportamentais e sugere a necessidade de atendimento psicológico ou psiquiátrico. Em quarto lugar, foram calculadas as freqüências e incidências de cada item da ECI nos dois grupos estudados, respectivamente. Em quinto lugar, foram analisados os subescores "neurótico" e "anti-social", de acordo com Graminha.

Em uma segunda etapa, após a preparação dos dados para análise, esses foram submetidos ao tratamento estatístico incluindo análises de estatística descritiva e inferencial. Foi realizada a análise comparativa entre os grupos PT e AT; na análise de variáveis contínuas foi utilizado o teste paramétrico $t$ para amostras independentes e na análise de variáveis discretas, o Teste Qui-Quadrado ou o Teste Exato de Fisher. Procedeu-se também à análise de correlação de Pearson entre os resultados no Raven, DFH e ECI do grupo PT, a fim de verificar a relação entre o desempenho nas áreas intelectual, emocional e comportamental das crianças. A análise de correlação será realizada apenas no grupo PT, para aprofundar a análise dos resultados do grupo de estudo. Foi verificada a relação entre gênero e resultado na ECI através do Teste Exato de Fisher. O valor do nível de significância adotado no presente estudo foi de $p=0,05$.

\section{Resultados}

Primeiramente, serão apresentados os resultados de comparação entre os grupos Pré-termo (PT) e A Termo (AT) e, em seguida, os de correlação entre a avaliação psicológica das crianças do grupo PT. A Tabela 1 apresenta as principais características das crianças e das mães dos grupos PT e AT.

As crianças do grupo PT tinham em média 8 anos e 9 meses de idade, enquanto as do grupo AT tinham em média 9 anos e 3 meses; verificou-se diferença significativa entre os grupos, embora esta represente uma pequena diferença em termos de meses e as crianças permaneçam na mesma etapa do desenvolvimento. Em relação à escolaridade, verificou-se que as crianças do grupo PT possuíam significativamente nível escolar mais atrasado em relação às crianças do grupo AT. A faixa de nível escolar de ambos os grupos situava-se de forma similar no primeiro ciclo do primeiro grau, sendo que as crianças PT encontravam-se em torno da $1^{\mathrm{a}}$ e $2^{\mathrm{a}}$ série e as crianças AT da $3^{\mathrm{a}}$ e $4^{\mathrm{a}}$ série do ensino fundamental.

Não foi detectada diferença significativa entre os grupos PT e AT quanto às características de escolaridade e nível ocupacional

Tabela 1

Características das Crianças dos Grupos Pré-termo (PT) e a Termo (AT) e das Respectivas Mães

\begin{tabular}{|c|c|c|c|}
\hline Variáveis & PT & AT & Valor de $p$ \\
\hline \multicolumn{4}{|l|}{ Idade da criança (meses) } \\
\hline Média $(d p)$ & $105,30( \pm 7,35)$ & $111,40( \pm 6,69)$ & \\
\hline $\begin{array}{l}\text { Escolaridade da criança } \\
\text { (Série) }(\%)\end{array}$ & & & $0,01 *$ \\
\hline $1^{\text {a }}$ série & $35 \%$ & 0 & \\
\hline $2^{a}$ série & $47 \%$ & 0 & $0,001 * *$ \\
\hline $3^{\mathrm{a}}$ série & $18 \%$ & $45 \%$ & \\
\hline $4^{a}$ série & 0 & $55 \%$ & \\
\hline \multicolumn{4}{|l|}{$\begin{array}{l}\text { Escolaridade da mãe - } \\
\text { (anos de escolaridade) }\end{array}$} \\
\hline Média $(d p)$ & $5,25( \pm 3,40)$ & $6,80( \pm 1,47)$ & \\
\hline \multicolumn{4}{|l|}{$\left(\right.$ Classificação $\left.^{1}\right)(\%)$} \\
\hline Do Lar & $50 \%$ & $47 \%$ & \\
\hline Qualificação Inferior & $15 \%$ & $38 \%$ & NS \\
\hline Não-qualificado & $35 \%$ & $15 \%$ & \\
\hline
\end{tabular}

Nota. $*$ Teste t ; ${ }^{* *}$ Teste Qui-quadrado; NS = Não-significativo; ${ }^{1}$ Classificação do nível ocupacional:

Qualificação Inferior; Não-qualificado (Soares \& Fernandes, 1989). 
Tabela 2

Comparação entre Resultados da Avaliação Psicológica em Diferentes Áreas do Desenvolvimento das Crianças dos Grupos Pré-termo (PT) e a Termo (AT)

\begin{tabular}{lccc}
\hline \multicolumn{1}{c}{ Áreas do } & PT & AT & Valor de $t$ \\
Desenvolvimento Psicológico da Criança & $m(d p)$ & $m(d p)$ & \\
\hline $\begin{array}{l}\text { Intelectual } \\
\text { (Raven - escore em percentil) }\end{array}$ & $37,00( \pm 24,25) *$ & $62,75( \pm 24,30)$ & 3,35 \\
$\begin{array}{l}\text { Evolutiva } \\
\text { (DFH- escore em percentil) }\end{array}$ & $55,80( \pm 36,10)$ & $57,25( \pm 28,26)$ & 0,14 \\
$\begin{array}{l}\text { Emocional } \\
\text { (DFH- escore em percentil) }\end{array}$ & $37,25( \pm 30,15)$ & $40,20( \pm 27,80)$ & 0,32 \\
$\begin{array}{l}\text { Comportamental } \\
\text { (ECI- escore em pontos) }\end{array}$ & $22,35( \pm 7,71)^{* *}$ & $14,75( \pm 10,12)$ & 2,67 \\
\hline
\end{tabular}

Nota. ${ }^{*} p=0,002 ; * * p=0,01$

Tabela 3

Escala Comportamental Infantil (ECI): Itens com Diferença Estatística Significativa entre os Grupos Pré-termo (PT) e a termo (AT)- Freqüencia (f) e Incidência (i)

\begin{tabular}{|c|c|c|c|c|c|}
\hline \multirow[t]{2}{*}{ Itens da ECI } & \multicolumn{2}{|c|}{$\begin{array}{c}\text { PT } \\
(n=20)\end{array}$} & \multicolumn{2}{|c|}{$\begin{array}{c}\mathrm{AT} \\
(n=20)\end{array}$} & \multirow[t]{2}{*}{$\begin{array}{l}\text { Direção da diferença entre grupos } \\
\text { (Teste Exato de Fisher) }\end{array}$} \\
\hline & $\mathrm{f}$ & i & $\mathrm{f}$ & i & \\
\hline Urina na cama & 4 & $0,20 *$ & 0 & 0 & $\mathrm{PT}>\mathrm{AT}$ \\
\hline Medo & 12 & $0,60 *$ & 6 & 0,30 & $\mathrm{PT}>\mathrm{AT}$ \\
\hline Movimento repetitivo, tique & 8 & $0,40^{*}$ & 2 & 0,10 & $\mathrm{PT}>\mathrm{AT}$ \\
\hline Agitada & 17 & $0,85^{* *}$ & 4 & 0,20 & $\mathrm{PT}>\mathrm{AT}$ \\
\hline Impaciente, irriquieta & 15 & $0,75^{*}$ & 6 & 0,30 & $\mathrm{PT}>\mathrm{AT}$ \\
\hline Não permanece nas atividades & 15 & $0,75^{*}$ & 8 & 0,40 & $\mathrm{PT}>\mathrm{AT}$ \\
\hline
\end{tabular}

Nota. ${ }^{*} p=0,05 ; * * p=0,001$

das mães; os indicadores sócio- educacionais do ambiente familiar de desenvolvimento de ambos os grupos eram equivalentes.

A Tabela 2 apresenta os resultados da avaliação psicológica dos grupos PT e AT, quanto a dimensões do desenvolvimento intelectual, emocional e comportamental.

Deve-se salientar que das 20 crianças PT avaliadas, duas (10\%) não conseguiram realizar o Raven devido à falta de compreensão das instruções padronizadas do teste. Estas crianças apresentaram história de graves seqüelas de comprometimento neurológico. Portanto, na comparação entre grupos com relação aos dados do Raven foi considerado o total de 18 crianças no grupo PT.

Os resultados da avaliação psicológica das crianças revelaram diferenças significativas entre os grupos PT e AT nas áreas de desenvolvimento relativas ao nível intelectual e indicadores de problemas comportamentais; as crianças PT apresentaram escores mais baixos no teste de Raven e escores mais altos na ECI em comparação às crianças do grupo AT.

As crianças PT apresentaram média dos percentis no Raven abaixo de 50, o que as classifica como "definidamente abaixo da média", considerando-se o grupo de padronização brasileira do teste. As crianças AT, por sua vez, apresentaram percentis no Raven abaixo da média. Com relação ao comportamento, as crianças PT, em comparação às crianças AT, apresentaram escore médio na ECI acima da nota de corte da amostra clínica equivalente a 16 pontos, estabelecida na padronização brasileira da escala. Portanto, as crianças PT apresentaram mais indicadores em nível clínico de problemas de comportamento do que as crianças AT (PT $=75 \% ; \mathrm{AT}=35 \%)$; esse achado sugere a necessidade de atendimento psicológico para a maior parte das crianças PT, de acordo com a recomendação do autor.

Não foi detectada diferença significativa entre o nível evolutivo e os indicadores emocionais avaliados por meio do DFH em ambos os grupos. Nos itens evolutivos, a média de ambos os grupos situou-se em torno do percentil 50, indicador de padrão de normalidade. Nos itens emocionais, por sua vez, ambos os grupos apresentaram médias em torno do percentil 40 , com leve tendência a apresentar problemas de ordem emocional em ambos os grupos.

A Tabela 3 apresenta os resultados dos itens da Escala de Comportamento Infantil A2 de Rutter que apresentaram diferença estatisticamente significativa entre os grupos PT e AT.

Observa-se na Tabela 3 que, segundo a opinião das mães, as crianças do grupo PT apresentaram significativamente mais do que as crianças do grupo AT os seguintes problemas de comportamento: enurese, medo, movimentos repetitivos ou tiques, agitação, impaciência ou inquietude e não permanência nas atividades.

$\mathrm{Na}$ análise dos subescores da ECI quanto aos tipos de comportamento internalizante (neurótico) e externalizante (antissocial) não foi detectada diferença significativa entre os grupos (PT $=27 \%$ [neurótico]; 27\% [antissocial] e AT $=29 \%$ [neurótico]; 29\% 
Tabela 4

Correlação entre os Resultados das Diferentes Áreas de Avaliação Psicológica do Grupo PT

\begin{tabular}{lllll}
\hline $\begin{array}{c}\text { Áreas do Desenvolvimento Psicológico } \\
\quad \text { (Medidas e Instrumentos) }\end{array}$ & 1 & 2 & 3 & 4 \\
\hline $\begin{array}{l}\text { 1- Nivel intelectual } \\
\text { (escore em percentil no Raven) }\end{array}$ & 1,00 & & & \\
$\begin{array}{l}\text { 2- Nível Evolutivo } \\
\text { (escore em percentil no DFH) }\end{array}$ & 0,43 & 1,00 & & \\
$\begin{array}{l}\text { 3- Indicadores Emocionais } \\
\text { (escore em percentil no DFH) }\end{array}$ & 0,19 & $0,55^{*}$ & 1,00 & \\
$\begin{array}{l}\text { 4- Comportamento } \\
\text { (escore em pontos na ECI) }\end{array}$ & $-0,40^{* *}$ & $-0,04$ & $-0,04$ & 1,00 \\
\hline
\end{tabular}

Nota. $* p=0,01 ; * * p=0,005$

[antissocial]; $\mathrm{p}=0,99)$. Além disso, comparando-se a amostra total subdividida quanto ao gênero, não foi encontrada diferença entre meninos e meninas com relação ao escore total da ECI em nível clínico $($ meninos $=53 \%$ e meninas $=56 \% ; p=0,56$ ).

A Tabela 4 mostra os coeficientes de correlação de Pearson entre os resultados das crianças do grupo PT na avaliação intelectual, obtidos através do teste de Raven, na avaliação dos indicadores evolutivos e emocionais, obtidos através do DFH, e na avaliação comportamental, através da ECI.

Verifica-se na Tabela 4 que, no grupo PT, houve correlação significativa negativa entre o nível intelectual medido através do teste de Raven e os indicadores de problemas de comportamento avaliados por meio da ECI, ou seja, quanto mais baixo o nível intelectual, mais problemas de comportamento nas crianças PT. Verificou-se também correlação significativa entre os indicadores evolutivos e emocionais medidos através do DFH, ou seja, quanto mais baixo o nível evolutivo mais indicadores sugestivos de problemas emocionais.

\section{Discussão}

A vulnerabilidade biológica decorrente da condição do nascimento pré-termo e do muito baixo peso ao nascer constituise em fator de alto risco para o desencadeamento de problemas de desenvolvimento em diferentes etapas evolutivas da trajetória de vida da criança. A avaliação psicológica, focalizando dimensões das áreas intelectual, emocional e comportamental, realizada na fase escolar de 20 crianças nascidas com menos de 37 semanas de idade gestacional e peso igual ou abaixo de 1.500 gramas (grupo PT), comparadas a 20 pares de crianças nascidas a termo (grupo AT) foi o objetivo do presente estudo.

Quanto à avaliação do nível intelectual dos dois grupos de crianças, constatou-se diferença estatística significativa entre o desempenho alcançado no teste psicométrico de Raven. As crianças nascidas prétermo e com muito baixo peso apresentaram resultados significativamente mais rebaixados em comparação aos obtidos pelas crianças nascidas a termo. Observou-se que, em média, as crianças do grupo PT classificaram-se no Raven, na zona limítrofe à faixa de inteligência média. Além disso, observou-se que as duas crianças restantes deste grupo não conseguiram realizar o teste de Raven, devido a problemas neurológicos graves, sugerindo desempenho intelectual deficiente. Por outro lado, o desempenho do grupo de crianças nascidas a termo mostrou ser superior ao do grupo de crianças pré-termo, considerando-se que as primeiras apresentaram nível intelectual na média ou acima da média.

Esses achados são consonantes aos resultados obtidos nos estudos de Taylor e colaboradores (1998) e Nadeau e colaboradores (2001), que utilizaram a avaliação psicométrica de inteligência e que demonstraram que quanto mais baixo o peso de nascimento da criança, mais chance de haver rebaixamento do nível intelectual. Smith e Knight-Jones (1990) e Smedler e colaboradores (1992) também verificaram que crianças pré-termo e muito baixo peso apresentavam escores significativamente mais rebaixados quando comparadas a grupo controle.

Forslund e Bjerre (1990) verificaram que crianças suecas prétermo e muito baixo peso, mesmo classificando-se dentro dos padrões normais na avaliação psicométrica e, portanto, sinalizando a presença de recursos cognitivos, obtiveram desempenhos mais rebaixados do que crianças nascidas a termo. Fitzhardinge e Steven (1972) demonstraram que mesmo apresentando nível intelectual médio, 50\% dos meninos e 36\% das meninas canadenses nascidos pré-termo e baixo peso apresentaram desempenho escolar insatisfatório.

Por outro lado, Mutch, Leyland e McGee (1993), Kalmar (1996) e Sansavini, Rizzard, Alessandroni e Giovanelli (1996) encontraram resultados mais otimistas em relação a essas crianças vulneráveis. Esses autores enfatizaram que não é só a condição de baixo peso e prematuridade que determina o desempenho cognitivo da criança. Outras variáveis podem concorrer simultaneamente, minimizando os efeitos dessa adversidade neonatal, que torna a criança vulnerável e contribui para que ela apresente desempenho satisfatório em procedimentos de mensuração de nível intelectual. Consonante com esses autores, no presente estudo, os resultados nos itens evolutivos no Desenho da Figura Humana revelou que o grupo PT apresentou indicadores de recurso intelectual em nível médio de forma semelhante ao grupo AT.

A diferença entre os resultados no Raven e no DFH pode ser entendida com base na diferença de habilidades cognitivas 
envolvidas nos respectivos testes. O Raven é um teste não-verbal que exige, segundo Sánchez (1987) raciocínio abstrato, discriminação, atenção seletiva, análise e síntese, pensamento analógico, entre outras operações. Essa tarefa cognitiva envolve, portanto, maior complexidade e abstração do que a exigida pelo DFH, que envolve a habilidade gráfica de representação do esquema corporal. Essa hipótese de maior dificuldade de execução do Raven em relação ao DFH confirma-se quando se observa que duas crianças do grupo PT não conseguiram realizar a tarefa solicitada no primeiro instrumento.

Salienta-se que no grupo de crianças PT estavam incluídas duas crianças portadoras de deficiência sensorial auditiva, sendo que uma delas apresentava deficiência grave. Este tipo de deficiência tem sido relatado por outros pesquisadores como seqüela importante em amostras de crianças nascidas pré-termo e baixo peso (Gyler, Dudley, Blinkhorn \& Barnett, 1993; Novello, Degraw \& Kleinnan, 1992; O’Brien, Soliday \& McCluskey-Fawcett, 1995).

Com relação aos aspectos emocionais das crianças do presente estudo, avaliados através dos itens emocionais do Desenho da Figura Humana, observou-se que ambos os grupos apresentaram sinais de problemas no funcionamento emocional, sugestivos pelo DFH de indicadores de ansiedade, preocupações e dificuldade em adotar estratégias adequadas de enfrentamento dessas dificuldades, quando comparadas com a amostra de padronização do referido teste psicométrico. Através do Desenho da Figura Humana, a criança projeta sua auto-imagem ou representação corporal, além de suas defesas e conflitos. O Desenho da Figura Humana reflete a personalidade do indivíduo e sua forma de interação com o meio ambiente (Cunha, 1993). A presença de indicadores emocionais nos desenhos das crianças que compuseram o grupo PT sugere problemas na área de representação corporal dessas crianças; porém, tais sinais não foram observados exclusivamente neste grupo.

Levy-Shiff e colaboradores (1994) verificaram que crianças pré-termo são mais propensas a apresentar uma série de dificuldades emocionais, mostrando-se menos adaptadas, além de apresentarem baixo autoconceito, do que crianças nascidas a termo. Notou-se, no entanto, no presente estudo que as crianças do grupo AT também apresentaram sinais de dificuldade emocional que podem advir de outras fontes que transcendem aos efeitos mediados pelas variáveis de risco neonatal.

Com relação à avaliação do comportamento das crianças, realizada através da Escala de Comportamento Infantil A2 de Rutter, considerando-se apenas o escore total obtido pelas crianças na escala, verificou-se que houve diferença estatística significativa entre os grupos PT e AT. A maioria das mães das crianças nascidas pré-termo e com muito baixo peso referiu alto índice de queixas comportamentais relativas aos seus filhos, em nível clínico sugestivo de indicação da necessidade de atendimento psicológico.

A comparação entre as incidências dos itens da ECI dos grupos PT e AT revelou diferenças estatisticamente significativas. As crianças do grupo PT, quando comparadas às crianças do grupo AT, mostraram maior incidência de problemas do tipo: enurese, medo, agitação, impaciência ou inquietude, movimentos repetitivos ou tiques e não permanência nas atividades. Os resultados confirmam os estudos de Stjernqvist (1993) e Levy-Shiff e colaboradores (1994), que observaram freqüência mais elevada de distúrbios de comportamento, tais como hiperatividade, comportamento opositor e inibição social entre crianças nascidas pré-termo e com muito baixo peso em comparação com crianças nascidas a termo.

No entanto, não foram verificadas diferenças entre os grupos com relação a padrões diferenciados de comportamento do tipo de internalização ou de externalização. Isso se confirma pelo fato de as crianças P'T apresentarem comportamentos tanto internalizantes sugestivos de ansiedade, tais como enurese, medo e tiques, quanto comportamentos externalizantes, tais como agitação, inquietude e não permanência em tarefas. Além disso, também não houve diferença significativa entre meninos e meninas quanto aos problemas de comportamento.

Os comportamentos de agitação, impaciência ou inquietude e não permanência nas atividades das crianças do grupo PT podem estar vinculados a um quadro de hiperatividade e agitação ou a um limiar rebaixado de tolerância à frustração das crianças em suas interações com o meio. Duas hipóteses poderiam explicar esse padrão comportamental. Primeiramente, práticas educativas inadequadas que podem não estar transmitindo à criança parâmetros necessários para a aquisição de regras e limites, provavelmente refletindo maior tolerância por parte das mães ao comportamento da criança, aliado ao excesso de proteção, como salientado anteriormente por Levy-Shiff e colaboradores (1994) e Sansavini e colaboradores (1996). Em segundo lugar, seqüelas do funcionamento neurológico decorrentes da condição de prematuridade e intercorrências neonatais, como encontrado por Taylor e colaboradores (1998).

$\mathrm{Na}$ amostra de crianças nascidas pré-termo com muito baixo peso estudada identificou-se um sub-grupo de crianças com comprometimento acentuado, tanto na esfera intelectual quanto na comportamental, que requer ajuda especializada educacional e terapêutica. Verificou-se neste grupo, que quanto mais ocorrem problemas intelectuais, mais ocorrem problemas de comportamento. A idade gestacional associada ao peso de nascimento, constitui-se, portanto, um fator relevante a ser considerado na formação de desenvolvimento futuro da criança, seja no âmbito cognitivo ou comportamental.

A prematuridade parece não ter relação direta com o desempenho futuro, porém demonstra forte relação como mediadora dos processos de desenvolvimento da criança. Devese salientar a influência potencial das variáveis proximais do ambiente familiar no desenvolvimento da criança, como por exemplo, as interações e mediações sociais, embora neste estudo não tenha sido objeto de análise. Kalmar (1996) e Bradley e colaboradores (1993) demonstraram que crianças pré-termo e muito baixo peso vivendo em condições de pobreza, mas experimentando um ambiente familiar com três ou mais fatores protetores (variedade da estimulação, suporte emocional, 
responsividade parental e aceitação do comportamento infantil) são mais propensas a apresentar sinais de resiliência do que crianças que não contam com esses mecanismos protetores. $\mathrm{O}$ ambiente assume, então, um papel importante e decisivo, na medida em que recursos externos podem ser mobilizados no sentido de promoverem mediação adequada a essas crianças, dando-lhes condições para a ativação de recursos que lhes permitirão um funcionamento cognitivo dentro dos parâmetros normativos satisfatórios.

\section{Considerações Finais}

A realização do presente estudo reuniu dados sobre indicadores do desenvolvimento psicológico de um grupo de crianças nascidas pré-termo com muito baixo peso de alto risco neonatal. Foram encontradas evidências de que as crianças nascidas pré-termo apresentaram desvantagens em indicadores desenvolvimentais relevantes por ocasião do marco evolutivo da fase escolar, quando comparadas a seus pares nascidos a termo.

Os resultados deste estudo foram sugestivos de achados importantes acerca do impacto da condição neonatal de vulnerabilidade sobre o desenvolvimento psicológico das crianças. Ficou evidenciada a necessidade de avaliação acerca das conseqüências de complicações neonatais sobre a trajetória de desenvolvimento da criança. Além da idade gestacional, o peso de nascimento e a evolução clínica do bebê na UTI Neonatal devem ser considerados e analisados enquanto fatores que podem estar ocorrendo simultaneamente entre as variáveis biológicas de risco.

Os achados sugerem a importância de programas de seguimento longitudinal do desenvolvimento das crianças nascidas em condição de risco, a fim de neutralizar precocemente as adversidades identificadas no desenvolvimento e comportamento antes do ingresso da criança na fase escolar. Deve-se considerar também a importância de se promover medidas educativas e/ou terapêuticas à parcela de crianças com comprometimentos moderados ou graves.

Deve-se, no entanto, destacar algumas limitações na interpretação dos resultados do presente estudo, a saber: a) os resultados do grupo PT restringem-se a uma amostra de crianças localizadas a partir do contexto hospitalar; b) houve grande perda de participantes na composição da amostra decorrente da dificuldade de localização e contato com as crianças após oito a dez anos do seu nascimento; c) as variáveis selecionadas para avaliar o desenvolvimento das crianças circunscrevem-se a variáveis da própria criança e a indicadores específicos de áreas do seu desenvolvimento, o que não contempla processos adaptativos complexos avaliados através da interação entre criança e o seu contexto de desenvolvimento.

Estudos futuros devem ser desenvolvidos no sentido de combinar a abordagem assistida de avaliação à avaliação psicométrica, e de incluir na avaliação, além de variáveis da própria criança, indicadores da interação desta com o ambiente promotor do seu desenvolvimento. Além disso, estudos com delineamentos longitudinais da trajetória de desenvolvimento das crianças nascidas pré-termo podem contribuir para identificação de processos de resiliência desencadeados em crianças vulneráveis devido a história neonatal de risco.

\section{Referências}

Angelini, A. L., Alves, I. C. B., Custódio, E. M. \& Duarte, W. F. (1987). ManualMatrizes Progressivas Coloridas de Raven (Escala Especial): Padronização Brasileira. São Paulo: Casa do Psicólogo.

Antunha, E. L. G. (1994). Avaliação neuropsicológica na infância (0 a 6 anos). Em V. B. de Oliveira \& N. A. Bossa (Orgs), Avaliação psicopedagógica da criança de zero a seis anos (pp. 89-122). Petrópolis, RJ: Vozes.

Bordin, M. B. M., Linhares, M. B. M. \& Jorge, S. M. (2001). Aspectos cognitivos e comportamentais na média meninice de crianças nascidas pré-termo e com muito baixo peso. Psicologia:Teoria e Pesquisa, 17, 49-57.

Bradley, R. H., Whiteside, L., Caldwell, B. M., Casey, P. H., Kelleher, K. \& Pope, S. (1993). Early indications of resilience and their relation to experiences in the home environments of low birthweight, premature children living in poverty. Child Development, 65, 346-360.

Brazelton, T. B. (1994). Momentos decisivos do desenvolvimento infantil (J. L. Camargo, Trad.). São Paulo: Martins Fontes. (Original publicado em 1992)

Callanan, C., Doyle, L. W., Rickards, A. L., Kelly, E. A., Ford, G. W. \& Davis, N. M. (2001). Children followed with difficulty: How do they differ? Journal of Paediatric Child Health, 37, 152-156.

Cockburn, F., Cooke, R. W. I., Gamsu, H. R., Greenough, A., Hopkins, A., Mcintosh, N., Agstont, S. A., Parryt, G. I., Silverman, M., Shaw, I. C. L., Tarnow-Modit, W. O. \& Wilkinson, A. R. (1993). The CRIB (Clinical Risk Index for Babies) score: A tool for assering inicial neonatal risk and comparing performance of neonatal intensive care units. Lancet, 342,193-198.

Cunha, J. A. (1993). Psicodiagnóstico - R. Porto Alegre: Artes Médicas.

Dammann, O., Walther, H.,Allers, B.,Schroder, M., Drescher, J., Lutz, D., Veelken, N. \& Schulte, F. J. (1996). Development of a regional cohort of very low birthweight children at six years: Cognitive abilities are associated with neurological disability and social background. Developmental Medicine and Child Neurology, 38, 97-108.

Fitzhardinge, P. M. \& Steven, E. M. (1972). The small-for-date infant II. Neurological and intellectual sequelae. Pediatrics, 50, 50-57.

Forslund, M. \& Bjerre, I. (1990). Follow-up of preterm children: II. Growth and development at four years of age. Early Human Development, 24, 107-118.

Girouard, P. C., Baillargeon, R. H., Tremblay, R. E., Glorieux, J., Lefebvre, F. \& Robaey, P. (1998). Developmental pathways leading to externalizing behaviors in 5 year old born before 29 weeks of gestation. Developmental and Behavioral Pediatrics, 19, 244-253.

Graminha, S. S. V. (1998). Recursos metodológicos para pesquisas sobre riscos e problemas emocionais e comportamentais na infância. Em G. Romanelli \& Z. M. M. Biasoli-Alves (Orgs), Diálogos metodológicos sobre práticas de pesquisa (pp. 71-87). Ribeirão Preto, SP: Legis Summa.

Grunau, R. E. (2000). Long-term consequences of pain in human neonates. Em K. J. S. Anand, B. J. Stevens \& P. J. McGrath (Orgs.), Pain in neonates (2 ${ }^{\text {nd }}$ ed.; pp. 55-76). Amsterdam: Elsevier Science.

Gyler, L., Dudley, M., Blinkhorn, S. \& Barnett, B. (1993). The relationship between psychosocial factors and developmental outcome for very low and extremely low birthweight infants: A review. Australian and New Zealand Journal of Psychiatry, 27, 62-73.

Hutz, C. S. \& Antoniazzi, A. S. (1995). O desenvolvimento da figura humana em crianças de 5 a 15 anos de idade: Normas para avaliação psicológica. Psicologia: Reflexão e Crítica, 8, 3-18.

Kalmar, M. (1996). The course of intellectual development in preterm and full term children: An 8-year longitudinal study. International Journal of Behavioral Development, 19, 491-516.

Langkamp, D. L., Kim, Y. \& Pascoe, J. M. (1998). Temperament of preterm infants at 4 months of age: Maternal rating and perceptions. Developmental and Behavioral Pediatrics, 19, 391-396.

Levy-Shiff, R., Einat, G., Har-Even, D., Mogilner, M., Mogilner, S., Lerman, M. \& Krikler, R. (1994). Emotional and behavioral adjustment in children born prematurely. Journal of Clinical Child Psychology, 23, 323-333.

Lewis, R. J., Duglokinski, E. L., Caputo, L. M. \& Griffin, R. B. (1988). Children at risk for emotional disorders: Risks and dimensions. Clinical Psychology Review, $8,417-440$. 
Liaw, F. R. \& Brooks-Gunn, J. (1993). Patterns of low-birth-weight children's cognitive development. Developmental Psychology, 29, 1024-1035.

Linhares, M. B. M., Carvalho, A. E. V., Bordin, M. B. M., Chimello, J. T., Martinez, F. E. \& Jorge, S. M. (2000). Prematuridade e muito baixo peso como fatores de risco ao desenvolvimento da criança. Cadernos de Psicologia e Educação Paidéia, 10(18), 60-69.

Lorenz, J. M. (2000). Survival of the extremely preterm infant in North America in the 1990s. Clinics of Perinatology, 27, 255-262.

Maas, Y. G., Mirmiran, M., Hart, A. A., Koppe, J. G., Ariagno, R. L. \& Spekreijse, H. (2000). Predictive value of neonatal neurological tests for developmental outcome of preterm infants. The Journal of Pediatrics, 137, 100-106.

Mutch, L., Leyland, A. \& McGee, A. (1993). Patterns of neuropsychological function in a low birthweight population. Developmental Medicine and Cbild Neurology, 35, 943-956.

Naudeau, L., Boivin, M., Tessier, R., Lefebvre, F. \& Robaey, P. (2001). Mediators of behavioral in 7-year-old children born after 24 to 28 weeks of gestation. Journal of Developmental and Behavioral Pediatrics, 22, 1-11.

Novello, A. C., Degraw, C. E. \& Kleinnan, D. V. (1992). Health children ready to learn: An essencial collaboration between health and education. Public Health Reports, 107, 3-10.

O’Brien, M., Soliday, E. \& McCluskey-Fawcett, K. (1995). Prematurity and the neonatal intensive care unit. Em M. C. Roberts (Org.), Handbook of pediatric psychology (2 $2^{\text {nd }}$. ed.; pp. 463-478). London: Guilford Press.

Piecuch, R. E., Leonard, C. H., Cooper, B. A. \& Sehring, S. A. (1997). Outcome of extremely low birth weight infants (500 to 999 grams) over a 12 -year period. Pediatrics, 100, 633-639.

Rutter, M. (1987). Psychosocial resilience and protective mechanisms. American Orthopsychiatric, 57, 316-331.
Sánchez, M. D. P. (1987). Elpotencial de aprendiraje: Un modelo y un sistema aplicado de evaluación. Murcia: Editora Universidad de Murcia.

Sansavini, A., Rizzard, M., Alessandroni, R. \& Giovanelli, G. (1996). The development of italian low and very low birthweight infants from birth to 5 years: The role of biological and social risks. International Journal of Behavioral Development, 19, 533-547.

Smedler, A. C., Faxelius, G., Bremme, K. \& Lagerstrom, M. (1992). Psychological development in children born with very low birthweight after severe intrauterine growth retardation: A 10-year follow-up study. Acta Paediatrica, 81, 197-203.

Smith, A. E. A. \& Knight-Jones, E. B. (1990). The habilities of very low-birthweight children and their classroom controls. Developmental Medicine and Child Neurology, 32, 590-601.

Soares, N. E. \& Fernandes, L. M. (1989). A medida do nível sócio-econômicocultural. Arquivos Brasileiros de Psicologia, 41, 35-43.

Stjernqvist, K. (1993). The early mother-infant interaction in the highly technological care environment. Acta Pediatrica, 82, 981-982.

Taylor, H. G., Klein, N., Schatschneider, C. \& Hack, M. (1998). Predictors of early school-age outcomes in very-low-birth-weight children. Journal of Developmental and Behavioral Pediatrics, 19, 235-243.

Waber, D. P. \& McCornick, M. C. (1995). Late neuropsychological outcomes in preterm infants of normal IQ: Selective vulnerability of the visual system. Journal of Pediatric Psychology, 20, 721-735.

Recebido: 17/07/2003

Última revisão: 09/02/2004

Aceite final: 08/03/2004

Sobre os autores

Maria Beatriz Martins Linhares é Psicóloga, Mestre pela Universidade Federal de São Carlos, Doutora pela Universidade de São Paulo, Pósdoutora pela University of British Columbia e pela Dalhousie University. É Professora da Universidade de São Paulo.

Juliana Thomazatti Chimello é Psicóloga, Mestranda pela Universidade de São Paulo.

Maria Beatriz Machado Bordin é Doutora pela Universidade de São Paulo.

Ana Emilia Vita Carvalho é Mestre e Doutora pela Universidade de São Paulo.

Francisco Eulógio Martinez é Mestre e Doutor pela Universidade de São Paulo e Pós-doutor pela University of British Columbia e pela University of Calgary. É Livre-docente na Universidade de São Paulo. 